\title{
An Adaptive Neural Network State Estimator for Quadrotor Unmanned Air Vehicle
}

\author{
Jiang Yuning ${ }^{1}$, Muhammad Ahmad Usman Rasool ${ }^{2}$, Qian Bo ${ }^{3}$, Ghulam Farid ${ }^{4}$, Sohaib Tahir Chaudary ${ }^{5}$ \\ State Grid Changzhou Power Supply Company, Changzhou, China ${ }^{1,3}$ \\ Shanghai Jiao Tong University, Shanghai, China ${ }^{2}$ \\ COMSATS University, Islamabad, Sahiwal Campus, 57000 Sahiwal, Pakistan ${ }^{4,5}$
}

\begin{abstract}
An adaptive neural observer design is presented for the nonlinear quadrotor unmanned aerial vehicle (UAV). This proposed observer design is motivated by the practical quadrotor where the whole dynamical model of system is unavailable. In this paper, dynamics of the quadrotor UAV system and its state space model are discussed and a neural observer design, using a back propagation algorithm is presented. The steady state error is reduced with the neural network term in the estimator design and the transient performance of the system is improved. This proposed methodology reduces the number of sensors and weight of the quadrotor which results in the decrease of manufacturing cost. A Lyapunov-based stability analysis is utilized to prove the convergence of error to the neighborhood of zero. The performance and capabilities of the design procedure are demonstrated by the Simulation results.
\end{abstract}

Keywords-Neural network observer; quadrotor; nonlinear systems; state estimator

\section{INTRODUCTION}

In recent years, quadrotor has become an interesting research area for the robotics community in the field of autonomous aerial vehicles. The application of hovering aerial vehicles have provided new opportunities to the researchers to find new control strategies for the better stabilization of the quadrotor. Mostly proposed approaches for autonomous aerial vehicles [1], [2] focused on the systems for an outdoor operation that could autonomously operate in the indoor environment and are also considered to be beneficial for the search and rescue operations. Unlike helicopters, quadrotor has movable blades and for changing the direction of rotation, quadrotor uses the rotational speed of its blades. This type of design provides flexibility in the movement of the quadrotor. The problem of estimating system state has already been done using Kalman filter [3], but the most important problem with the design procedure of classic observers is the presence of external disturbance and unknown dynamical model. In this paper the wind is considered as a disturbance factor. In the past studies, researchers have eliminated the wind effects by applying Robust and Adaptive controllers such as nonlinear adaptive feedback controller in [4], and terminal sliding mode controller is designed in [5] to stabilize the quadrotor system.

The accurate measurements of the system states, such as position, altitude, and velocity of quadrotor are critical, so this work has applied a neural observer to achieve more accurate approximation of system states in presence of the disturbance. Many researches have been carried out on neural and fuzzy controllers/observers in discrete time systems [6], [7]. Various control approaches have been applied on quadrotor such as machine learning [8], and feedback linearization with highorder sliding mode observer for the quadrotor [9]. In [10], a new Neural Network Observer (NNO) is designed to estimate the translational and angular velocities of the UAV, and an output feedback control law is developed in which the position and the attitude of the UAV are considered as a state variable to control the aircraft more accurately. In [11], a new dynamic neural network based observer is presented and is proved using sliding mode stability analysis so in the presence of uncertainty, disturbance and sensor noise it could asymptotically track the states of a quadrotor and blade flapping. A recurrent neuro-adaptive observer for a general model of MIMO nonlinear systems is presented in [12], where the stable observer is nonlinear in parameters. The network weights are updated based on a combination of a modified Back Propagation algorithm and an e-modification that guarantees the boundedness of the state estimation error. In [13], the attitude and altitude control of quadrotor UAV, and the application of Neural Network based on Direct Inverse Control (DIC) is proposed. The backpropagation learning algorithm [14] is utilized in order to find the appropriate connection weights of neurons by using real quadrotor flight data in hovering state.

In this work, a neural observer is designed to estimate the trajectory of the nonlinear quadrotor. Some terms in the dynamic model of quadrotor was unknown and also wind as a disturbance was added to the structure of quadrotor. In order to solve this problem, this work has applied a back-propagation algorithm to update the weights adaptively and also to eliminate the effect caused by external disturbance. Here, the point is that this work has considered the whole dynamic of quadrotor model undefined and the result shows the capability of neural network in the prediction and estimation of nonlinear functions.

The structure of this paper is organized as follows. Section II, introduces the dynamics of quadrotor system. In Section III, the neural estimator is described. The stability proof is presented in Section IV. In Section V, we validate the neural estimator for quadrotor via simulation results. Finally concluding remarks are presented in Section VI. 


\section{DYNAMICS OF QUADROTOR SYSTEM}

The dynamics of quadrotor system are shown:

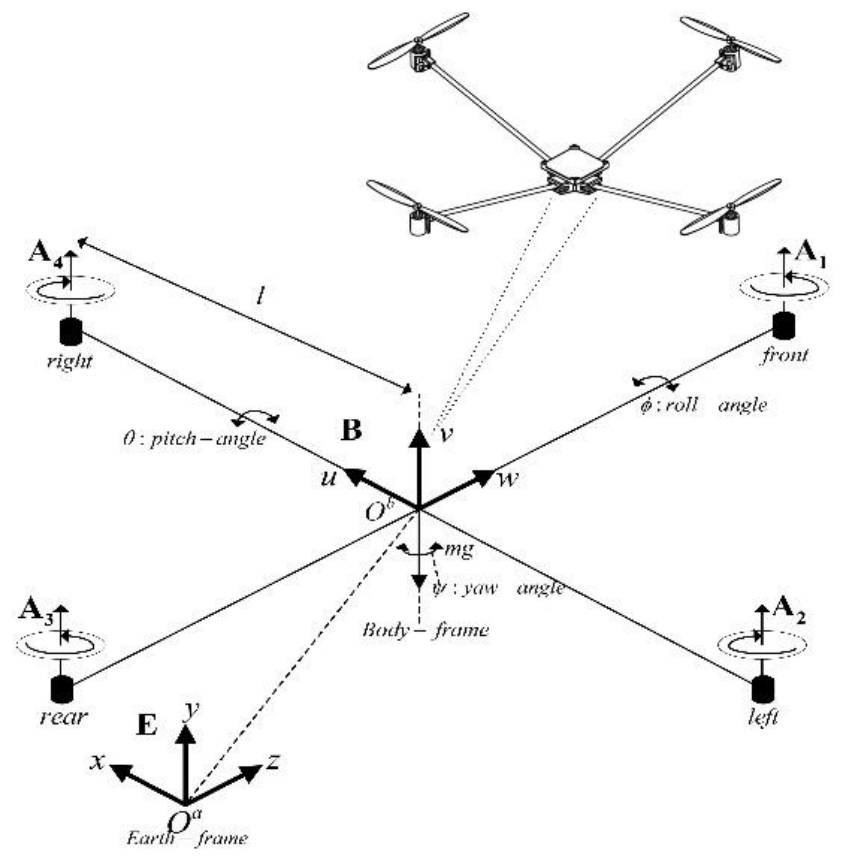

Fig. 1. Schematics of Quadrotor Forces.

The model of the quadrotor in this paper is set up by the body-frame $B$ and Earth-frame $E$ as represented in the Fig. 1. Let the forces on the quadrotor is represented as $A^{1}, A^{2}, A^{3}, A^{4}$ its vector $[u, v, w]^{\prime}$ denotes the position of the center of gravity of the quadrotor in the body frame, the vector $[x, y, z]^{\prime}$ denotes the linear velocity in the earth-frame, $m$ denotes the total mass, $g$ represents the acceleration of gravity, and $l$ denotes the distance from the center of each rotor to the center of gravity of quadrotor. The orientation of the quadrotor is given by the rotation matrix $R: E \rightarrow B$, where $R$ depends on the yaw, pitch and roll angles (Euler's Angle) which is represented as $[\psi, \theta, \phi]$, respectively. By using the transformation matrices and rotation matrices, the equations of quadrotor dynamical model is transferred to control standard model as follows:

$$
\left\{\begin{array}{l}
\dot{x}_{1}=x_{2} \\
\dot{x}_{2}=\left(\cos x_{7} \sin x_{9} \cos x_{11}+\sin x_{7} \sin x_{11}\right) \frac{u_{1}}{m}-\frac{k_{1} x_{2}}{m} \\
\dot{x}_{3}=x_{4} \\
\dot{x}_{4}=\left(\cos x_{7} \sin x_{9} \sin x_{11}+\sin x_{7} \cos x_{11}\right) \frac{u_{1}}{m}-\frac{k_{2} x_{4}}{m} \\
\dot{x}_{5}=x_{6} \\
\dot{x}_{6}=\left(\cos x_{7} \cos x_{9}\right) \frac{u_{1}}{m}-g-\frac{k_{3} x_{6}}{m} \\
\dot{x}_{7}=x_{8} \\
\dot{x}_{8}=-x_{10} x_{12} \frac{I_{y}-I_{z}}{I_{x}}+\frac{L}{I_{x}} u_{2}-\frac{k_{4} L}{I_{x}} x_{8}
\end{array}\right.
$$

$$
\left\{\begin{array}{l}
\dot{x}_{9}=x_{10} \\
\dot{x}_{10}=-x_{8} x_{12} \frac{I_{z}-I_{x}}{I_{y}}+\frac{L}{I_{y}} u_{3}-\frac{k_{5} L}{I_{y}} x_{10} \\
\dot{x}_{11}=x_{12} \\
\dot{x}_{12}=-x_{8} x_{10} \frac{I_{x}-I_{y}}{I_{z}}+\frac{1}{I_{z}} u_{4}-\frac{k_{6}}{I_{y}} x_{12}
\end{array}\right.
$$

where $x_{i}(i=1, \ldots ., 12)$ are the system states and $x_{1}, x_{3}, x_{5}$ are the quadrotor gravity center in the direction of $x, y, z$. The components $x_{2}, x_{4}, x_{6}$ are the speed along the direction of $x, y, z$ and $I_{x}, I_{y}, I_{z}$ represent the initial torque along the direction of $x, y, z$. On the other hand, $x_{7}, x_{9}, x_{11}$ shows the angles of roll, pitch, and yaw whereas $x_{8}, x_{10}, x_{12}$ show the torque of roll, pitch and yaw. $u_{i}=(i=1, \ldots ., 4)$ is the system input, and $k_{i}=(i=1, \ldots ., 6)$ represents the stretch coefficient.

\section{Neural Network State Estimator}

The structure of neural observer is shown in Fig. 2.

The neural network used in this paper has a hidden layer. For the output layer, this work has used linear activation function and the weights of the output layer are considered constant, whereas, the Sigmoid activation function is applied in the hidden layer:

$$
\phi_{(x, u)}=\frac{1}{1+\mathrm{e}^{-(x, u)}}
$$

Conditions of the activation function [15], in neural network are i) continuous; ii) derivable to its function; iii) capable of saturation to asymptotically approach to its maximum and minimum values; iv) applicable for a nonlinear system.

From Kolmogorov theorem every nonlinear function with any degree of complexity could be rewrite as an activation function and the weights:

$$
g(x, u)=w^{T} \phi(x, u)+\varepsilon(x)
$$

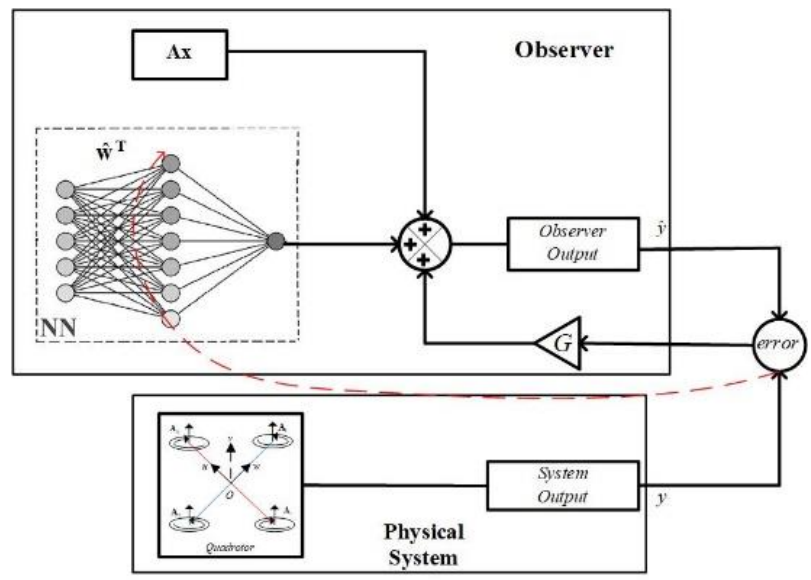

Fig. 2. Structure of Neural Observer. 
where, $w^{T}$ is the weight of the hidden layer, $g$ represents the unknown part of the quadrotor system, $\varepsilon(x)$ represents the neural network error.

Here, the quadrotor system with external disturbances could be described as follows:

$\dot{x}=F(x, u)+d(x)$

$y=C x$

The states and inputs of the quadrotor system are $x=x_{i}(i=1, \ldots ., 12)$ and $u=u_{i}(i=1, \ldots ., 12)$ respectively, and $d(x)$ represents the disturbance of the system.

By adding and subtracting $A x$ in equation (4), the following equation could be written as:

$\dot{x}=A x+g(x, u)$

$y=C x$

From the above equation, $A$ represents the optional matrix which should be Hurwitz and must be taken in such a way that the pair $(A, C)$ is observable:

$g(x, u)=F(x, u)-A x+d(x)$

The Luenburger observer structure is as follows [16]:

$\hat{\dot{x}}=A \hat{x}+\hat{g}(\hat{x}, u)+G(y-\hat{y})$

$\hat{y}=C \hat{x}$

where $\hat{x}$ is the observer state and $G$ is the observer gain. The observer gain should be selected such that Eigenvalues of the $A-G C$ will be Hurwitz.

Therefore, we consider the error as follows:

$\tilde{x}=x-\hat{x}$

By taking differential from the equation (8), and adding and subtracting $w^{T} \phi(\hat{x}, u)$ we get:

$\tilde{\dot{x}}=A_{c} \tilde{x}+\tilde{w}^{T} \phi(\hat{x}, u)+w^{T}[\phi(x, u)-\phi(\hat{x}, u)]+\varepsilon(x)$

where, $\tilde{w}=w-\hat{w}$ and $A_{c}=A-G C$.

For defining the neural network weights, we consider the cost function as follows:

$J=\frac{1}{2} \tilde{y}^{T} \tilde{y}$

where, $\tilde{y}=y-\hat{y}$. The modified error back propagation algorithm is defined as:

$\dot{\hat{w}}=-\eta \frac{\partial J}{\partial \hat{w}}-\rho\|\tilde{y}\| \hat{w}$

Where, $\eta$ is the learning rate and $\rho$ is the damping coefficient. The estimation of the unknown function could be written as: $\hat{g}(x, u)=\hat{w}^{T} \phi(\hat{x}, u)$

By adapting the chain rule, we have:

$\frac{\partial J}{\partial \hat{w}}=\frac{\partial j}{\partial \tilde{y}} \cdot \frac{\partial \tilde{y}}{\partial \tilde{x}} \cdot \frac{\partial \tilde{x}}{\partial \hat{g}(\hat{x}, u)} \cdot \frac{\partial \hat{g}(\hat{x}, u)}{\partial \hat{w}}=\tilde{y}^{T} C \frac{\partial \tilde{x}}{\hat{g}(\hat{x}, u)} \phi(\hat{x}, u)$

By using the equation (9), we have:

$\frac{\partial \dot{\tilde{x}}}{\partial \hat{g}(\hat{x}, u)}=(A-G C) \frac{\partial \tilde{x}}{\partial \hat{g}(\hat{x}, u)}-I \rightarrow \frac{\partial \tilde{x}}{\partial \hat{g}(\hat{x}, u)} \approx A_{c}^{-1}$

We could define the update law by the equation (15):

$\dot{\hat{w}}=-\eta \phi(\hat{x}, u)\left(\tilde{y}^{T} C A_{c}^{-1}\right)-\rho\|\tilde{y}\| \hat{w}$

And the weights error is defined as:

$\dot{\tilde{w}}=\eta \phi(\hat{x}, u)\left(\tilde{y}^{T} C A_{c}^{-1}\right)+\rho\|\tilde{y}\| \hat{w}$

\section{Stability Proof}

In order to prove the stability of the observer, we need to ensure the stability of the error dynamics and update law. For this, we have applied Lyapunov direct method. Considering the following Lyapunov candidate function:

$V=\frac{1}{2} \tilde{x}^{T} p \tilde{x}+\frac{1}{2} \operatorname{tr}\left(\tilde{w}^{T} \tilde{w}\right)$

Here, $p$ is the positive definite matrix which satisfies the following condition:

$A_{c}^{T} p+p A_{c}=-Q$

$Q$ is the positive definite matrix. By differentiation of the Lyapunov function, we get:

$\dot{V}=\frac{1}{2} \tilde{x}^{T} p \dot{\tilde{x}}+\frac{1}{2} \dot{\tilde{x}}^{T} p \tilde{x}+\operatorname{tr}\left(\tilde{w}^{T} \dot{\tilde{w}}\right)$

Substituting, equation (9) and (16) into equation (19) we conclude that:

$\dot{V}=-\frac{1}{2} \tilde{x}^{T} Q \tilde{x}+\tilde{x}^{T} p\left[\tilde{w}^{T} \phi(\hat{x}, u)+\omega(t)\right]$

$+\operatorname{tr}\left(\tilde{w}^{T} \eta \phi(\hat{x}, u)\left(\tilde{y}^{T} C A_{c}^{-1}\right)+\tilde{w}^{T} \rho\|\tilde{y}\| \hat{w}\right.$

where,

$\omega_{(t)}=w^{T}(\phi(x, u)-\phi(\hat{x}, u))+\varepsilon(x)$

Satisfying the following inequality, we get:

$\operatorname{tr}\left(\tilde{w}^{T}(w-\hat{w}) \leq w_{M}\|\tilde{w}\|-\|\tilde{w}\|^{2}\right.$

$\operatorname{tr}\left(\tilde{w}^{T} \phi(\hat{x}, u) \tilde{x}^{T} l_{1}\right) \leq \phi_{M}\|\tilde{w}\|\|\tilde{x}\|\left\|l_{1}\right\|$

Putting, $l_{1}=\eta C^{T} C A_{c}^{-1}$ in the equation (20), we get: 
$\dot{V} \leq-\frac{1}{2} \lambda_{\min } Q\|\tilde{x}\|^{2}+[\|x\|\|p\|\|\tilde{w}\| \phi(\hat{x}, u)+\bar{\omega})$

$+\phi_{M}\|\tilde{w}\|\|\tilde{x}\|\left\|l_{1}\right\|+\left[w_{M}\|\tilde{w}\|-\|\tilde{w}\|^{2}\right] \rho\|C \tilde{x}\|$

Now, considering $N_{1}=\frac{\left\|l_{1}\right\|}{2}$, then adding and subtracting $N_{1}^{2}\|\tilde{w}\|^{2}\|\tilde{x}\|$, we get:

$\dot{V} \leq-\frac{1}{2} \lambda_{\min } Q\|\tilde{x}\|^{2}+\left[\|p\| \bar{w}-\|\tilde{w}\| \rho\|C\|-N_{1}^{2}\right)$

$\left.+\|\tilde{w}\|\left(\|p\| \phi_{M}+\phi_{M}\left\|l_{1}\right\|+w_{M} \rho\|C\|\right)-N_{1}^{2}\|\tilde{w}\|^{2}\right]\|\tilde{x}\|$

Here, we have $N_{2}$ as follows:

$N_{2}=\frac{\|P\| \phi_{M}\left\|l_{1}\right\|+w_{M} \rho\|C\|}{2\left(\rho\|C\|-N_{1}^{2}\right)}$

Now, by adding and subtracting $N_{2}^{2}\|\tilde{x}\|$, gives the following results:

$\dot{V} \leq-\frac{1}{2} \lambda_{\min } Q\|\tilde{x}\|^{2}+\|\tilde{x}\|\left[\|p\| \bar{\omega}+\left(\rho\|C\|-N_{1}^{2}\right) N_{2}^{2}\right.$

$\left.-\left(\rho\|C\|-N_{1}^{2}\right)\left(N_{2}-\|\tilde{w}\|\right)^{2}-\left(N_{1}\|w\|\right)^{2}\right]\|\tilde{x}\|$

The obtained result would be negative if the following condition is satisfied:

$\|\tilde{x}\|>\frac{2}{\lambda_{\min } Q}\left[\|p\| \bar{\omega}+\left(\rho\|C\|-N_{1}^{2}\right) N_{2}^{2}\right]$

$\rho \geq \frac{N_{1}^{2}}{\|C\|}$

From the above equations, the result shows that error of system and weight are limited, therefore observer remains stable.

\section{Simulation Results}

To analyze the performance of the observer, the first step is to stabilize the system with a sliding mode controller. In the second step, we apply the observer on the quadrotor system to show the effectiveness of the design procedure. Here, we suppose the initial condition for quadrotor states as [ $\begin{array}{lll}0.1 & 0.2 & 0.3\end{array}$ 0.40 .50 .40 .100 .50 .40 .60 .7 . Consider the matrix $\mathrm{A}$ and $\mathrm{C}$ as follows:

$$
\begin{aligned}
A & =\left[\begin{array}{ccccc}
0.001 & 1 & & 0 \\
& & 0.001 & \ddots & \\
& & & \ddots & 1 \\
0 & & & & 0.001
\end{array}\right]_{12 \times 12} \\
C & =\left[\begin{array}{llllllllllll}
1 & 0 & 0 & 0 & 0 & 0 & 0 & 0 & 0 & 0 & 0 & 0 \\
0 & 0 & 1 & 0 & 0 & 0 & 0 & 0 & 0 & 0 & 0 & 0 \\
0 & 0 & 0 & 0 & 0 & 0 & 1 & 0 & 0 & 0 & 0 & 0 \\
0 & 0 & 0 & 0 & 0 & 0 & 0 & 0 & 1 & 0 & 0 & 0
\end{array}\right]
\end{aligned}
$$

TABLE I. PARAMETERS OF QUADROTOR SYSTEM AND OBSERVER

\begin{tabular}{|c|l|c|}
\hline Parameter & Value & Unit \\
\hline$m$ & 2 & $\mathrm{~kg}$ \\
\hline$L$ & 0.2 & $\mathrm{~m}$ \\
\hline$I x=I y$ & 1.2 & $\mathrm{Ns} / \mathrm{rad}$ \\
\hline$I z$ & 2.2 & $\mathrm{Ns^{2 }} / \mathrm{rad}$ \\
\hline$J r$ & 0.2 & $\mathrm{Ns} / \mathrm{rad}$ \\
\hline$K 1, K 2, K 3$ & 0.01 & $\mathrm{Ns} / \mathrm{m}$ \\
\hline$K 4, K 5, K 6$ & 0.012 & $\mathrm{Ns} / \mathrm{m}$ \\
\hline$g$ & 9.8 & $\mathrm{~m} / \mathrm{s}^{2}$ \\
\hline$b$ & 5 & $\mathrm{Ns} / \mathrm{m}$ \\
\hline$d$ & 2 & $\mathrm{~N} / \mathrm{ms}$ \\
\hline$\rho$ & 0.001 & - \\
\hline$\eta$ & 3000 & - \\
\hline
\end{tabular}

The parameters of quadrotor system and observer are given in Table 1. Here, the point is to distinguish the error between the practical system and estimated system by adopting the proposed method. By simulation we obtain the results as shown in the following figures:
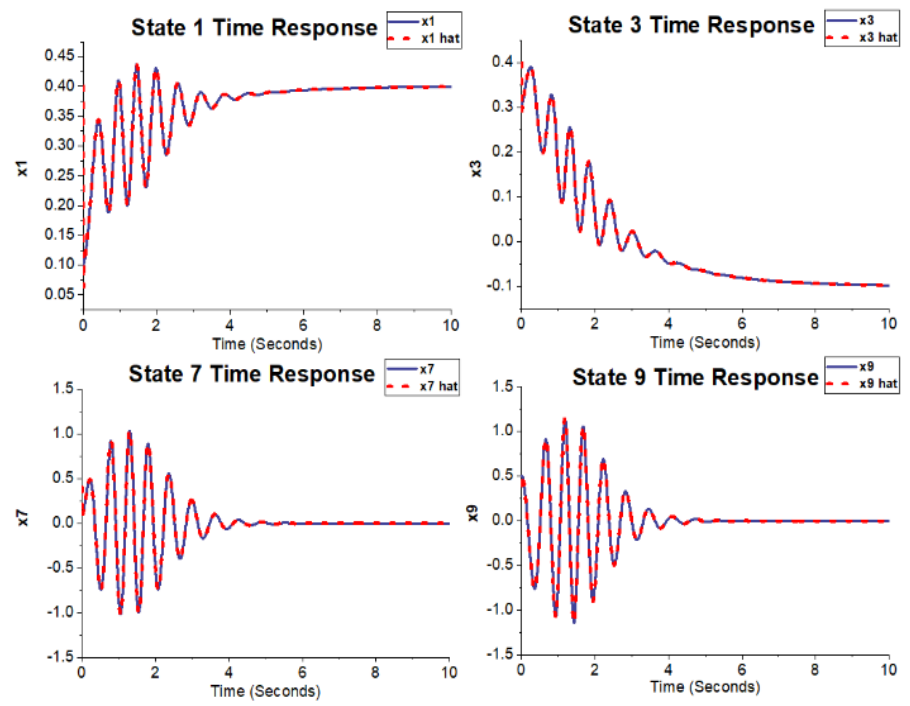

Fig. 3. State 1, 3, 7, 9 and Estimated State Trajectory of Quadrotor.

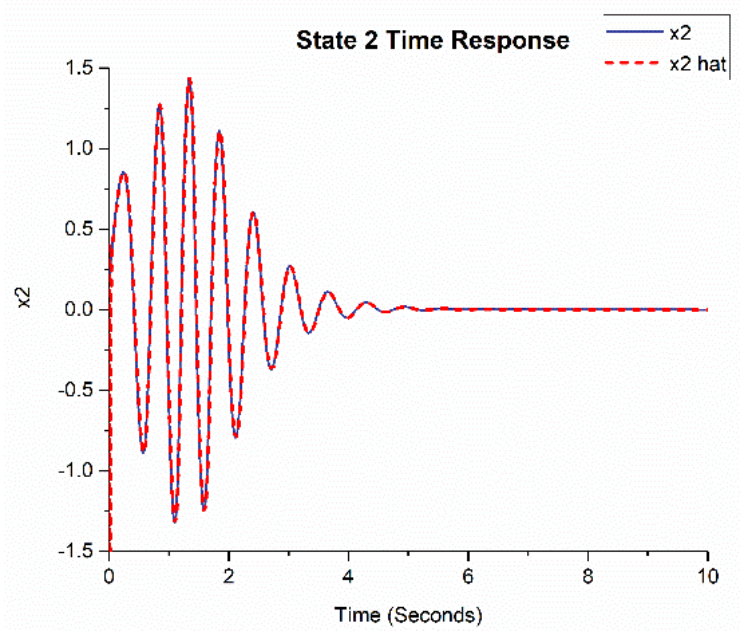

Fig. 4. State 2 and Estimated State Trajectory of Quadrotor. 


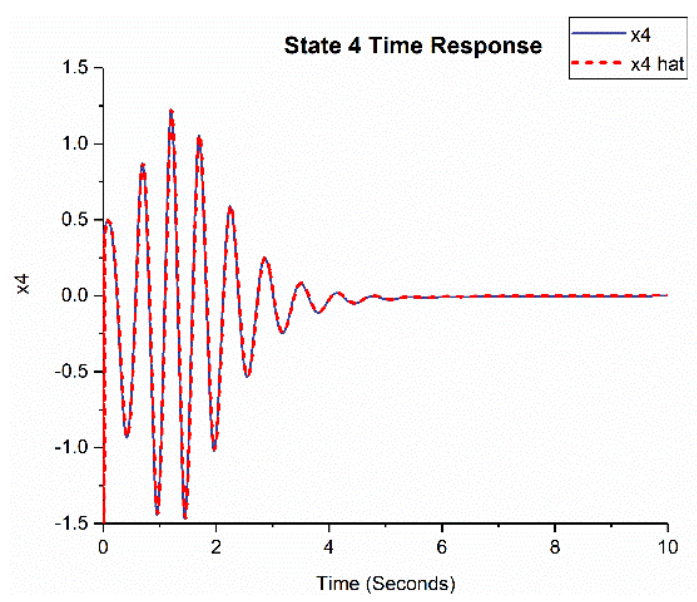

Fig. 5. State 4 and Estimated State Trajectory of Quadrotor.

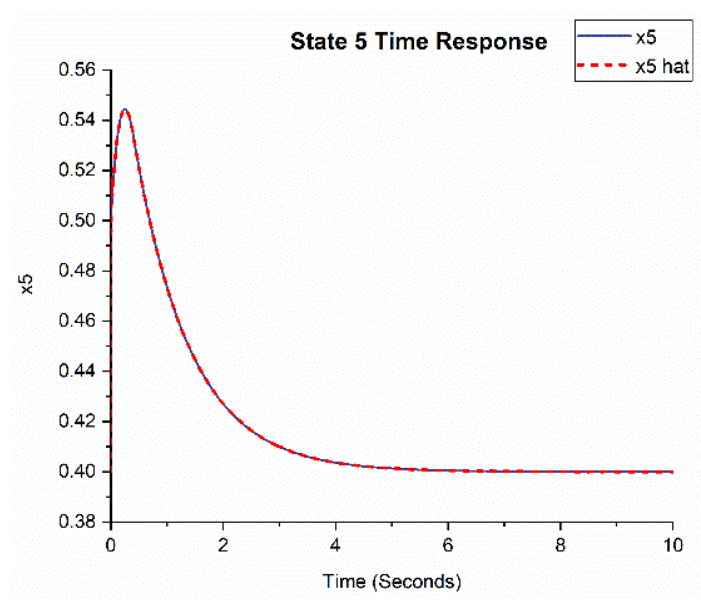

Fig. 6. State 5 and Estimated State Trajectory of Quadrotor.

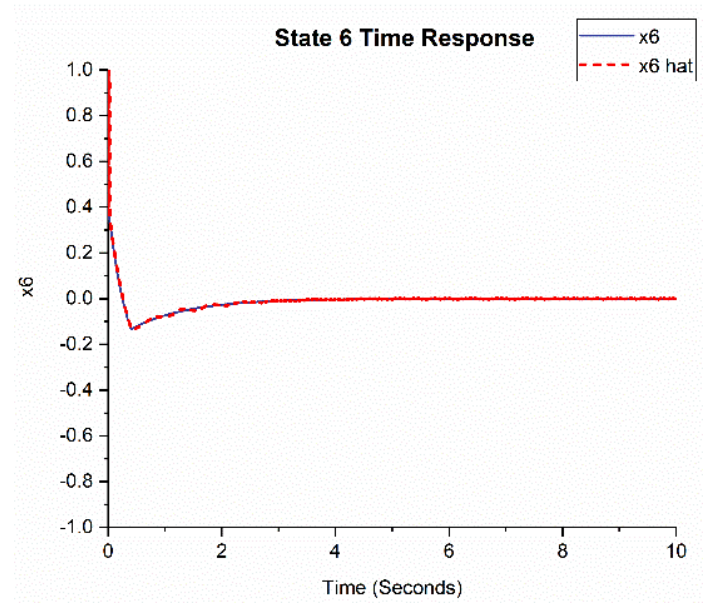

Fig. 7. State 6 and Estimated State Trajectory of Quadrotor.

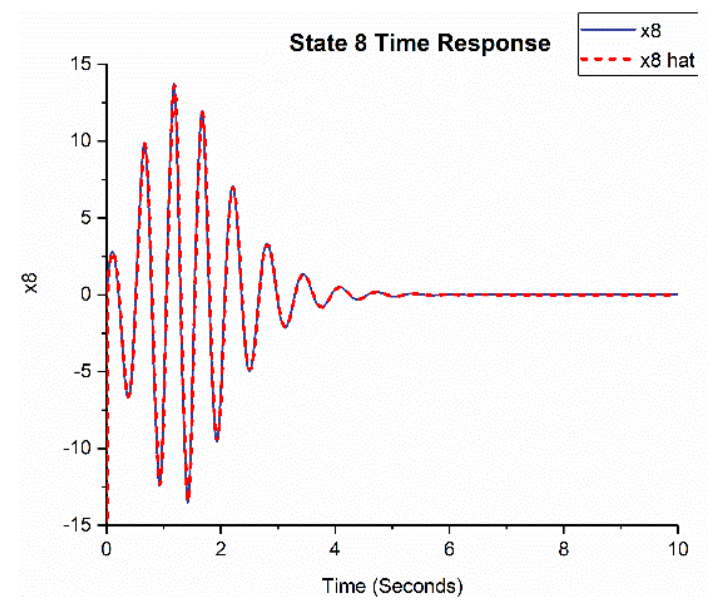

Fig. 8. State 8 and Estimated State Trajectory of Quadrotor.

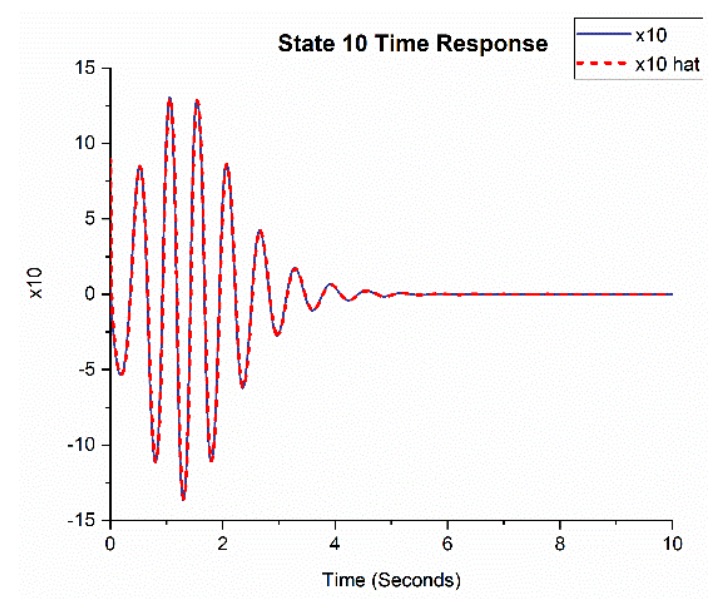

Fig. 9. State 10 and Estimated State Trajectory of Quadrotor.

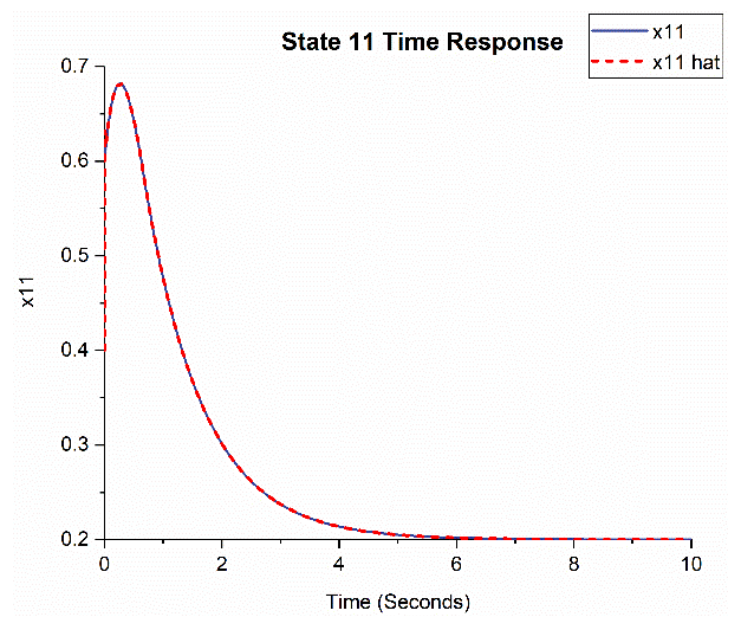

Fig. 10. State 11 and Estimated State Trajectory of Quadrotor. 


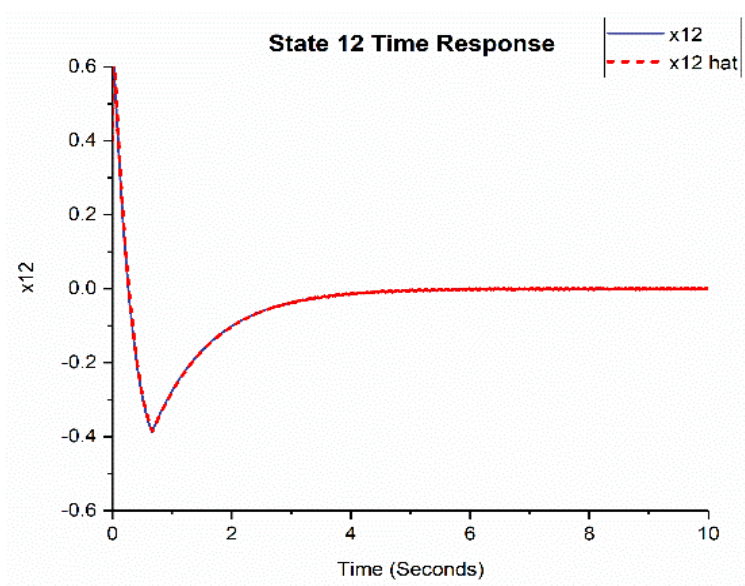

Fig. 11. State 12 and Estimated State Trajectory of Quadrotor.

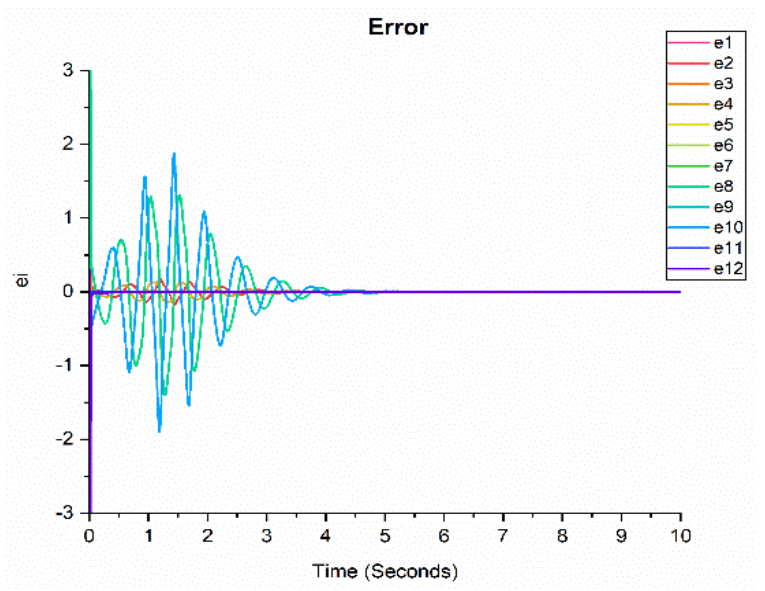

Fig. 12. Error between Observer States and Quadrotor System.

The states $\mathrm{x}_{1}, \mathrm{x}_{3}, \mathrm{x}_{7}$, and $\mathrm{x}_{9}$ are presented in the output of the system and the error between observer estimation and quadrotor system is negligible and it is shown in Fig. 3, that the observer has properly estimated the practical system states $\mathrm{x}_{1}$, $\mathrm{x}_{3}, \mathrm{x}_{7}$ and $\mathrm{x}_{9}$. Fig. 4, 5, 6, 7, 8, 9, 10 and 11 illustrated that the observer effectively estimate the trajectory of the practical system states $\mathrm{x}_{2}, \mathrm{x}_{4}, \mathrm{x}_{5}, \mathrm{x}_{6}, \mathrm{x}_{8}, \mathrm{x}_{10}, \mathrm{x}_{11}$ and $\mathrm{x}_{12}$ respectively, which are not presented in the output. Although, these states are not presented in the output, the observer could distinguish state trajectory of the system. Fig. 12 shows that all errors gradually decreases over time and reaches to the neighborhood of zero. Moreover due to the use of adaptive structure in the design procedure the observer properly distinguish and eliminate the external disturbance.

\section{CONCLUSION}

This paper discussed a new approach to design an adaptive neural observer for the estimation of the nonlinear dynamics of quadrotor. The proper estimation of the practical system states, robustness against noise, disturbance, and the convergence of tracking error to the neighborhood of zero are the main advantages of this proposed method. It is evident from the MATLAB/SIMULINK results, that the proposed method could effectively predict the system behavior and eliminate the effect caused by the external disturbance. The stability of the overall system was shown by Lyapunov stability analysis. The design procedure results in the decrement of the number of sensors, weight of quadrotor and manufacturing costs which in turn increases the battery life. In future, this method can be expanded for more than one hidden layer of the neural network and it also can be applied to power or a natural systems.

\section{REFERENCES}

[1] Zeng, Y., Zhang, R., \& Lim, T. J. (2016). Wireless communications with unmanned aerial vehicles: Opportunities and challenges. IEEE Communications Magazine, 54(5), 36-42.

[2] Hayat, S., Yanmaz, E., \& Muzaffar, R. (2016). Survey on unmanned aerial vehicle networks for civil applications: A communications viewpoint. IEEE Communications Surveys \& Tutorials, 18(4), 26242661.

[3] Xiong, J. J., \& Zheng, E. H. (2015). Optimal kalman filter for state estimation of a quadrotor UAV. Optik, 126(21), 2862-2868.

[4] Xian, B., Diao, C., Zhao, B., \& Zhang, Y. (2015). Nonlinear robust output feedback tracking control of a quadrotor UAV using quaternion representation. Nonlinear Dynamics, 79(4), 2735-2752.

[5] Lu, Q., Ren, B., Parameswaran, S., \& Zhong, Q. C. (2018). Uncertainty and Disturbance Estimator-Based Robust Trajectory Tracking Control for a Quadrotor in a Global Positioning System-Denied Environment. Journal of Dynamic Systems, Measurement, and Control, 140(3), 031001.

[6] Cervantes, J., Muñoz, F., González-Hernández, I., Salazar, S., Chairez, I., \& Lozano, R. (2017, June). Neuro-fuzzy controller for attitudetracking stabilization of a multi-rotor unmanned aerial system. In Unmanned Aircraft Systems (ICUAS), 2017 International Conference on (pp. 1816-1823). IEEE.

[7] Yu, L., Chen, J., Tian, Y., Sun, Y., \& Ding, L. (2017). Fuzzy logic algorithm of hovering control for the quadrotor unmanned aerial system. International Journal of Intelligent Computing and Cybernetics, (justaccepted), 00-00.

[8] Choi, S., Kim, S., \& Kim, H. J. (2017). Inverse reinforcement learning control for trajectory tracking of a multirotor UAV. International Journal of Control, Automation and Systems, 15(4), 1826-1834.

[9] Fethalla, N., Saad, M., Michalska, H., \& Ghommam, J. (2018). Robust observer-based dynamic sliding mode controller for a quadrotor UAV. IEEE Access, 6, 45846-45859.

[10] Dierks, T., \& Jagannathan, S. (2010). Output feedback control of a quadrotor UAV using neural networks. IEEE transactions on neural networks, 21(1), 50-66.

[11] Heryanto, M., Suprijono, H., Suprapto, B. Y., \& Kusumoputro, B. (2017). Attitude and Altitude Control of a Quadcopter Using Neural Network Based Direct Inverse Control Scheme. Advanced Science Letters, 23(5), 4060-4064.

[12] Zhou, Y., Chen, M., \& Jiang, C. (2015). Robust tracking control of uncertain MIMO nonlinear systems with application to UAVs. IEEE/CAA Journal of Automatica Sinica, 2(1), 25-32.

[13] Luenberger, D. (1971). An introduction to observers. IEEE Transactions on automatic control, 16(6), 596-602.

[14] Jia, J., \& Duan, H. (2017). Automatic target recognition system for unmanned aerial vehicle via backpropagation artificial neural network. Aircraft Engineering and Aerospace Technology, 89(1), 145-154.

[15] Schmidhuber, J. (2015). Deep learning in neural networks: An overview. Neural networks, 61, 85-117.

[16] Taha, W., Al-Durra, A., Errouissi, R., \& Al-Wahedi, K. (2018, October). Nonlinear Disturbance Observer-Based Control for Quadrotor UAV. In IECON 2018-44th Annual Conference of the IEEE Industrial Electronics Society (pp. 2589-2595). IEEE. 